\title{
STABILITY OF MODULATED BEAM TRANSPORT IN RELATIVISTIC KLYSTRON TWO-BEAM ACCELERATORS*
}

\author{
S.M. Lidia, S.S. Yu, Lawrence Berkeley National Laboratory, Berkeley, CA 94720 USA
}

\section{Abstract}

We discuss the stability of the drive beam in a relativistic klystron two-beam accelerator (RK-TBA). Simulations are presented for the main extraction section of an RKTBA. Longitudinal and transverse equilibria are discussed. Beamline parameters are chosen which correspond to the RTA experiment.

\section{INTRODUCTION}

The relativistic klystron two beam accelerator (RK-TBA) concept [1] is actively being pursued by experimental and theoretical programs at LBNL [2], and CEA/CESTA [3]. The LBNL effort is concerned with building a prototype, the RTA [4], to physics, engineering, and costing issues in a full-scale device from the source and injector, accelerator, modulator, and power extraction sections. The RTA is designed to produce $\sim 180-200 \mathrm{MW} / \mathrm{m}$ of microwave power from standing wave rf cavities in the main extraction section. Kinetic energy in the beam is replaced by the use of highly efficient induction acceleration modules. Transverse focusing is provided by a permanent magnet FODO lattice. Longitudinal focusing is provided by inductively detuning the rf output structures. The FODO lattice is designed to produce a half-betatron oscillation between adjacent $\mathrm{rf}$ cavities, to minimize the effects of a cumulative dipole BBU mode associated with the cavities. A schematic of a single period of the main extraction section is shown in Figure 1.

In this paper, we discuss the beam dynamics in the main extraction section of the RTA. In particular, we examine the results of numerical simulation using the 3-D, timedependent RKS code [5]. We have modeled the beam dynamics and the evolution of the cavity fields from initial transients into the steady-state regime. The main parameters of the simulation are given in Table 1.

\section{EVOLUTION IN LONGITUDINAL PHASE-SPACE}

The longitudinal dynamics of the rf bunches constitute the heart of any RK-TBA. The energy extracted from the bunches in the rf cavities must be replaced by the induction modules. The detuning of the cavity introduces a nonlinear correlation between energy and phase for particles within the bunch. This induces the bunch to rotate in longitudinal

\footnotetext{
* This work supported under the auspices of the U.S. Department of Energy by LBNL under Contract No. AC03-76SF00098.
}

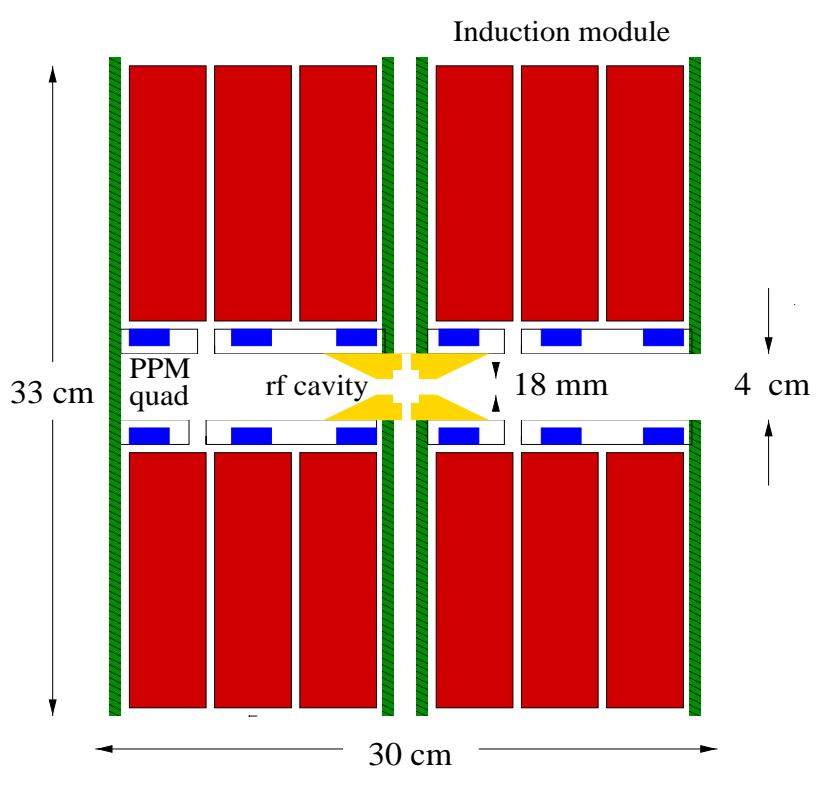

Figure 1: One period of the main RTA extraction section.

\begin{tabular}{|c|c|}
\hline Nominal beam energy & $3 \mathrm{MeV}$ \\
\hline \hline Beam current, dc & $600 \mathrm{~A}$ \\
\hline$\epsilon_{\text {edge }}$, normalized & $800 \pi \mathrm{mm} \mathrm{mr}$ \\
\hline Bunching parameter & 0.84 \\
\hline FODO cell length & $10 \mathrm{~cm}$ \\
\hline Phase advance/cell & $60 \mathrm{deg}$. \\
\hline Induction gap voltage & $45 \mathrm{kV}$ \\
\hline Modulation frequency & $11.424 \mathrm{GHz}$ \\
\hline Rf cavity frequency & $11.55 \mathrm{GHz}$ \\
\hline$Q_{\text {loaded }}$ & 56 \\
\hline$[\mathrm{R} / \mathrm{Q}]$ & $10 \Omega$ \\
\hline Total simulation length & $6 \mathrm{~m}$ \\
\hline
\end{tabular}

Table 1: Simulation parameters. 


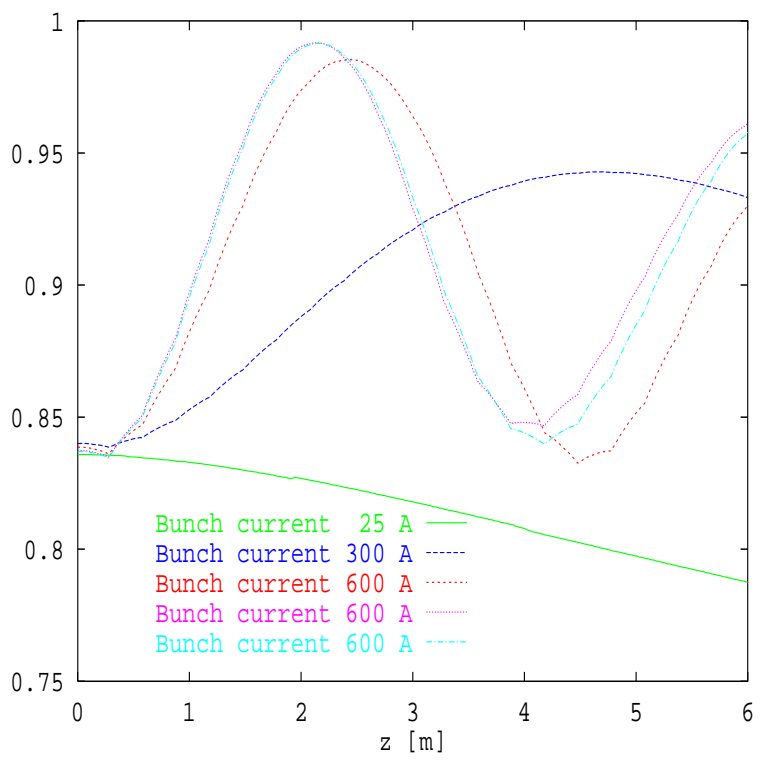

Figure 2: Bunching parameter variation along beamline.

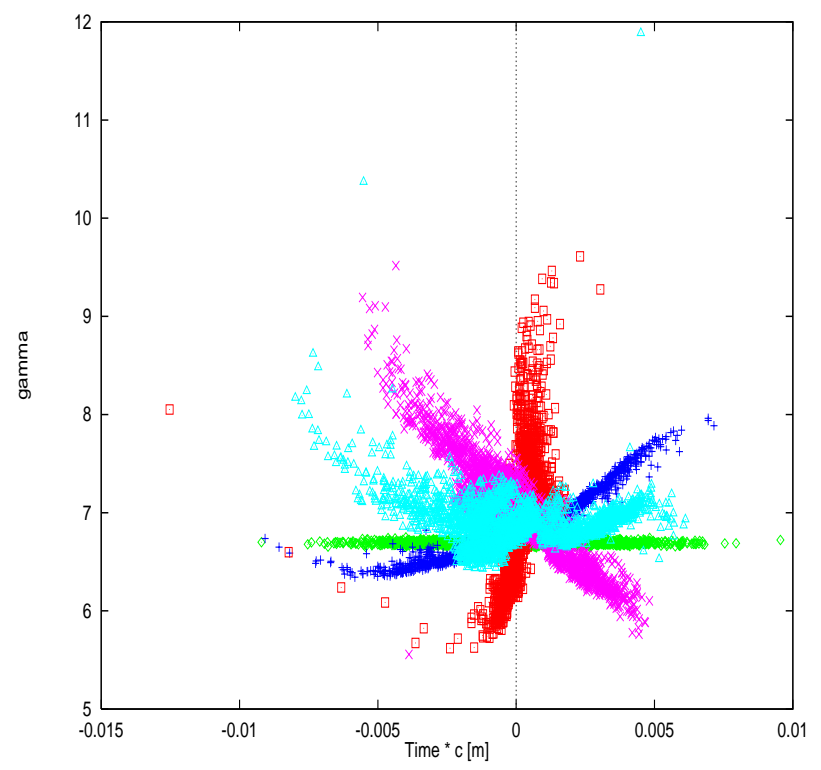

Figure 3: Bunch rotation along the beamline.

phase space. The re-acceleration in the induction modules is weakly dependent upon the dc beam current, but the rf cavity field excitation is strongly dependent upon both the $\mathrm{dc}$ current and the bunching parameter, $\mathrm{b}\left(\simeq \frac{1}{2} I_{r f} / I_{d c}\right)$. In our simulations, we see that while the dc current carried by a given bunch remains relatively constant, the bunching parameter varies significantly along the beamline, as well as temporally during the initial current ramp of the beam head. In Figure 2, we show the variation of the bunching parameter along the beamline, for bunches of lower dc current in the beam head through bunches in the main body of the beam. This clearly shows the synchrotron oscillation that follows the bunch rotation, shown in Figure 3.

We also see another effects of inductive detuning. Low

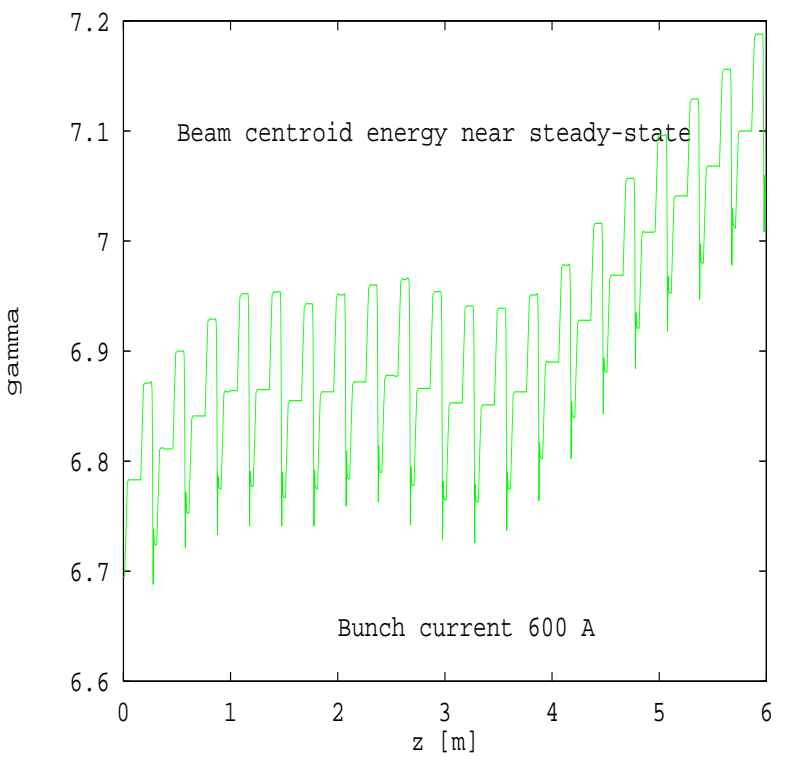

Figure 4: Variation of bunch centroid energy along beamline.

current, moderately compressed bunches resonantly excite cavity modes that react upon later bunches, which become more highly compressed. Due to their smaller bunch length, these later bunches will excite stronger fields in cavities further down the beamline, losing more energy as they do so. This is shown in Figure 4.

Later along in the synchrotron oscillation, these later bunches have a longer bunch length and deposit less energy into the cavity modes. Hence, they lose less energy in traversing a cavity. Inductive detuning thus has two manifestations in the bunch dynamics: a rotation in the longitudinal phase space of the bunch; and an oscillation in the bunch centroid energy. These oscillations affect the coupling of the bunch to the rf cavities, resulting in an oscillation along the beamline of the output power levels of the cavities, shown in Figure 5.

\section{EVOLUTION IN TRANSVERSE PHASE-SPACE}

The behavior of the bunches in longitudinal phase space effect the transverse dynamics as well. In particular we notice that an initially matched transport system, loses that feature as the bunch compresses and the bunch energy spread increases. The effect of bunch compression is to enhance the peak radial, defocusing self-fields in the presence of the conducting beampipe, while the enhanced energy spread introduces lower energy particles into the transport lattice. These two effects can, in principle, be dealt with by appropriately adjusting the strength of the quadrupoles along the beamline. In our studies we have maintained a constant parameter FODO lattice. In Figure 6 we show the evolution of the two transverse rms envelopes along the beamline for bunches in the center of the beam. 


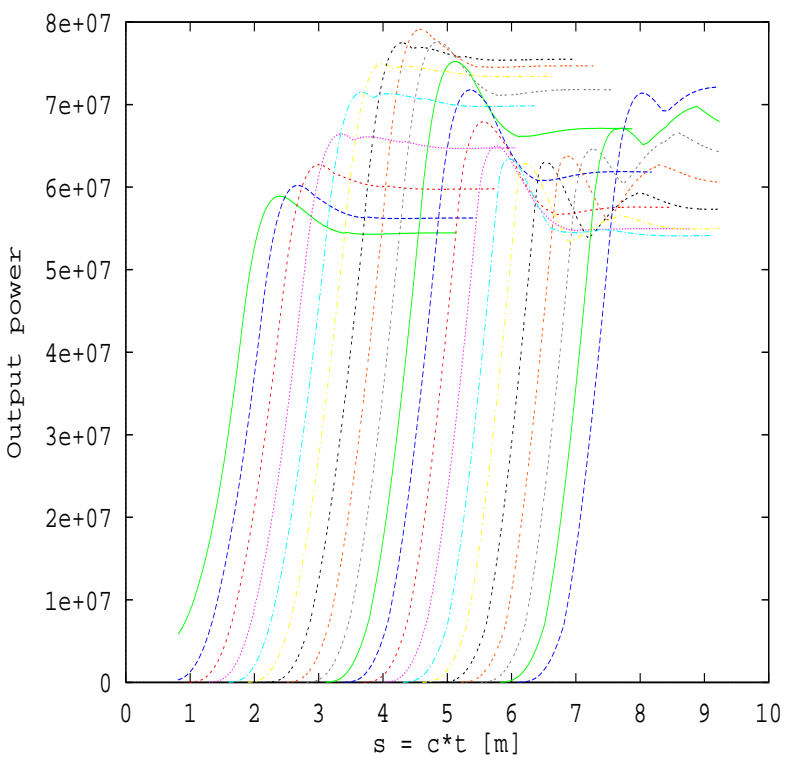

Figure 5: Power output from 20 rf cavities.

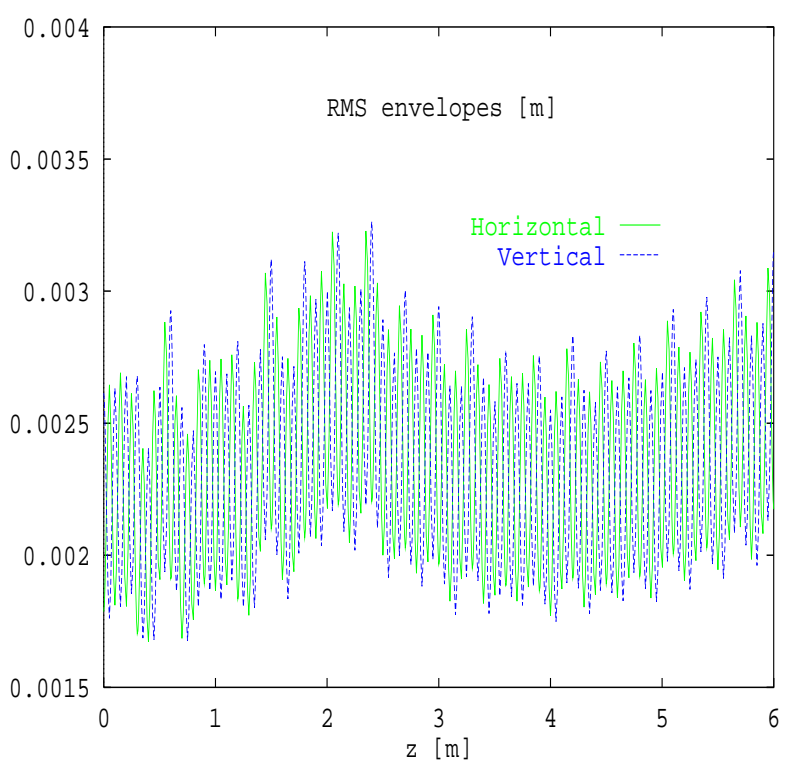

Figure 6: Evolution of transverse rms envelopes .

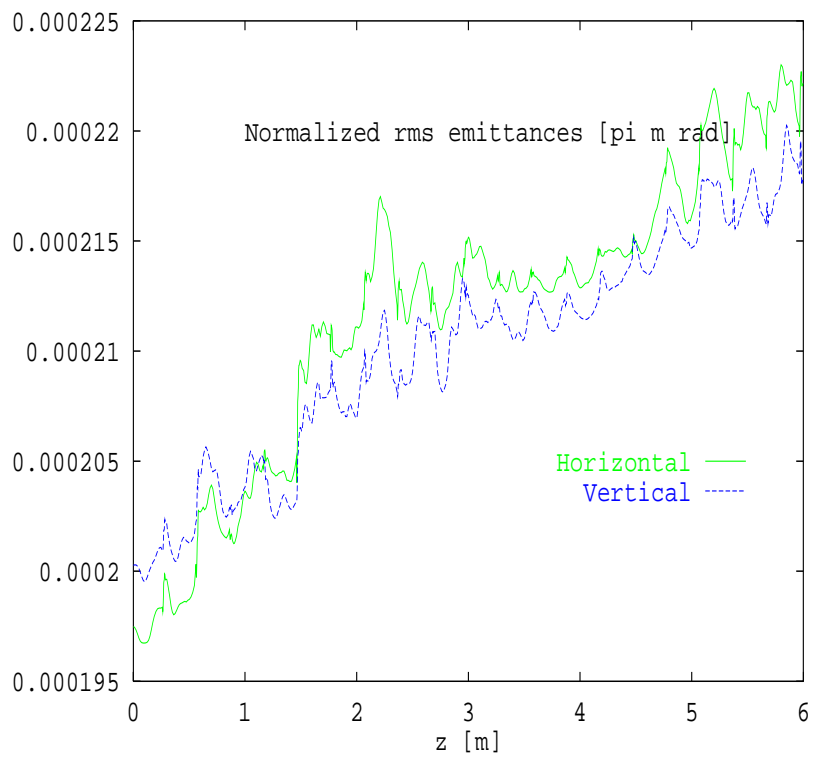

Figure 7: Evolution of transverse emittance.

In Figure 7, we show the evolution of the normalized rms transverse emittance. These simulations do not include the influence of transverse dipole modes in the induction modules or rf cavities. Thus, we have not included the important BBU instabilities that have shown to limit current transport in RK-TBA's. However, this simulation shows that pure rf monopole modes, 3-D space charge effects, and quadrupole transport do not significantly increase the transverse emittance in the beam. On average, we see these effects contributing an increase of $\sim 0.5 \%$ /TBA period.

\section{CONCLUSIONS}

We have presented simulations of beam transport and the evolution in longitudinal phase space in an RK-TBA system. Longitudinal stability has been shown to result from inductively detuning the output rf structures. The coupling between longitudinal and transverse phase spaces has been shown to result in only a modest growth rate of the transverse emittance.

\section{REFERENCES}

[1] A. Sessler and S. Yu. Relativistic klystron two-beam accelerator. Phys. Rev. Lett., 54:889, 1985.

[2] T. Houck et. al. Prototype microwave source for a relativistic klystron two-beam accelerator. IEEE Trans. Plasma Sci., 24(3):938, 1996.

[3] T. Lefevre et. al. Results on the interaction of an intense bunched electron beam with resonant cavities at $35 \mathrm{ghz}$. In these proceedings, 1999.

[4] S. Lidia et. al. Initial commissioning results of the rta injector. In these proceedings, 1999.

[5] S. Lidia. Relativistic klystron simulator. Unpublished, 1999. 